\title{
Photo-controllable percolation of decorated nanoparticles in a nanopore: molecular dynamics simulation study
}

\author{
Ilnytskyi J. ${ }^{1,2,3}$, Slyusarchuk A. ${ }^{2}$, Saphiannikova M. ${ }^{3}$ \\ ${ }^{1}$ Institute for Condensed Matter Physics of the Nat. Acad. Sci. of Ukraine, \\ 1 Svientsitskii Str., 79011 Lviv, Ukraine \\ ${ }^{2}$ Lviv Polytechnic National University, 12 S. Bandera str., 79013, Lviv, Ukraine \\ ${ }^{3}$ Leibniz Institute of Polymer Research, 6 Hohe Str., 01069, Dresden, Germany
}

(Received 10 June 2016)

\begin{abstract}
By means of molecular dynamics simulations we study formation of the wall-to-wall percolation cluster in the solution of decorated nanoparticles in a pore. The model takes into account photo-switching between unpolar trans and polar cis-isomers of azobenzene. This leads to either colloidal dispersion of the particles or their aggregation in a polar solvent. The dynamics of percolation cluster formation is analysed by applying a pulse-like illumination. We found that the wall-to-wall percolation is subject to the competition between nanoparticles aggregation, wall adsorption and microphase separation of the nanoparticles.
\end{abstract}

Keywords: gold nanoparticles, azobenzene, percolation.

2000 MSC: $82 \mathrm{C} 05,82 \mathrm{C} 43$

UDC: 538.9

\section{Motivation}

The nanoparticles decorated by certain functional groups are very important from the point of view of their optical, electronic, magnetic and chemical properties [1]. The applications to mention are: drug delivery, nanoelectronics, information storage, sensors, photocatalysis, development of smart materials, etc. [2]. Each particular application demands either good dispersion of such decorated nanopaticles or, vice versa, their micellization, aggregation or self-assembly. Of special importance is controlled self-assembly, by means of certain external stimulus. One of the ways to provide such control is by light (see, e.g. Ref. [3]). To this end the chromophoric (e.g. azobenzene, cinnamoyl, diarylethene dithiophenols, etc.) functional groups are incorporated into the decoration shell of a nanoparticle. Examples of applications include: photocontrollable micellisation $[4,5]$, optically switchable devices $[6,7]$, networks of nanoparticles with optically switchable conductance [8], etc.

Photocontrollable self-assembly requires certain special conditions. The first one: there should be enough free volume around chromophores to be able to photoisomerise. For instance, such condition is not satisfied for the densely grafted monolayers (involving azobenzene) on a planar gold, but the condition is met for the case of curved surface of gold nanoparticle of the size of $2 \mathrm{~nm}[9,10]$. The second condition is related to the resonance energy transfer between the electrons of a gold nanopaticle and of the azobenzene. This effect is essentially suppressed by the increase of the length of a polymer spacer between the nanoparticle and a chromophore [11]. It was found that the photoisomerisation of chromophores requires a spacer of at least $l \sim 12$ methylene units. The third condition for the photocontrollable aggregation or self-assembly of nanoparticles is the difference between the properties of the two isomers of the chromophore such as, for instance, their solubility in a particular solvent.

Photoisomerisation part of the study was supported by DFG grant GR 3725/2-2, molecular network part - by FP7 EU IRSES projects No. 612707 and 612669 
We will restrict our study to the case of azobenzene-based chromophores. They exist in two states of trans- and cis-isomer, which are different in a number of ways (see, e.g. [12]). In particular, (a) transisomer is mesogenic (can form liquid crystalline phases) and cis-isomer is not; (b) the dipole moment of trans and cis-isomer could have very different magnitude. The difference in a liquid crystallinity (a) is related to a prolate shape of trans-isomer and bended shape of cis-isomer, and is so far less explored for technological application. The difference in their dipole moment magnitude (b) already found a number of application. In particular, in the case of 4-alkoxyazobenzene (particular type of the azobenzene-based chromophore), the dipole moment of trans-isomer is about 1D, whereas it is about 5D for the cis-isomer [13]. Therefore, gold nanoparticles functionalised by such chromophores will be dispersed under visible (Vis) light in a hydrophobic solvent (e.g. cyclohexane). Upon irradiation with the ultraviolet (UV) light, the chromophores photoisomerise into polar cis-form and a rapid aggregation of the nanoparticles is observed [13]. Similar effect was reported for the nanoparticles in another hydrophobic solvent, toluene [14].

In contrary, in a polar solvent an opposite effect is observed. The nanoparticles aggregate under Vis light and disperse upon UV illumination [15]. This was reported for the case of a $1: 3$ chloroformethanol mixture, where gold nanoparticles capped with cis-isomers of substituted azobenzene were maintained in a dispersed state and those capped with trans-isomer coagulated. Similar effect is also reported by Raimondo et al. [16], they considered gold nanoparticles of three sizes randing from $3 \mathrm{~nm}$ to $30 \mathrm{~nm}$ coated by biphenyl type of azobenzene chromophore. In a toluene, the nanoparticles form a colloidal dispersion when chromophores are in a cis form, or aggregate when these switch to the transform. In this context there is a number of studies related to the photo-switchable nanoconductors based either on a special type of chromophores forming a percolating network between two walls-electrodes [8], or based on the composite materials comprising the mixture of chromophores and cabon nanotubes [17]. Therefore, the properties of the aggregates of decorated nanoparticles, and the possibility of percolation cluster formation, are of great technological importance.

The aim of the current paper is to model the dynamics of nanoparticles aggregation when confined within a nanopore with modified walls. The model for a decorated nanoparticle has been suggested and studied earlier [18-20] for the case of no illumination. The explicit trans-cis photoisomerisation for a coarse-grained model of azobenzene is discussed in a recent paper [21]. Here we combine both approaches. The modification of the walls promotes attraction to them the trans-isomers of the nanoparticles shell and, as the result, adsorption of the nanoparticles. The aggregation/deaggregation of nanoparticles inside a bulk of a pore is controlled by the choice of interaction potentials. According to the difference (a) between the trans- and cis-isomers (see, above), the trans-trans interaction is set to be attractive, whereas cis-cis and trans-cis are soft repulsive. The difference (b) between the isomers is reflected in their respective interaction with a polar solvent: trans-solvent pairwise interaction is repulsive, whereas cis-solvent - attractive. More details on a model and simulation approach are provided in Sec.2, main results concerning the formation of a percolation cluster are to be found in Sec. 3, conclusions are provided in Sec. 4 .

\section{The model}

The coarse-grained model for a decorated nanoparticle used in this study is the same as in a number of previous papers [18-20]. It comprises a spherical core bead functionalised by $N_{\text {ch }}$ chains each terminated by a chromophore, see Fig. 1 . The diameter of a core bead is chosen to be $2.14 \mathrm{~nm}$, providing high enough curvature of its surface to avoid the effect of steric hindrance for the chromophores [9]. Each spacer consists of 4 smaller soft spherical beads of the diameters $0.623 \mathrm{~nm}$ (the one adjacent to the core bead) and $0.459 \mathrm{~nm}$ (the rest ones). Each spacer bead represents a group of approximately 3 hydrocarbons, resulting in an equivalent spacer length of about 12 hydrocarbons. According to Ref. [11], at this spacer length a negligible suppression of the chromophores photoisomerisation takes place relatively to the case of free chromophores. Both trans and cis-chromophores are modelled as

Mathematical Modeling and Computing, Vol. 3, No. 1, pp. 33-42 (2016) 
soft spherocylinders of the same breadth of $D=0.374 \mathrm{~nm}$ and the elongation of $L / D=3$ but differ by means of their pair interaction potential.

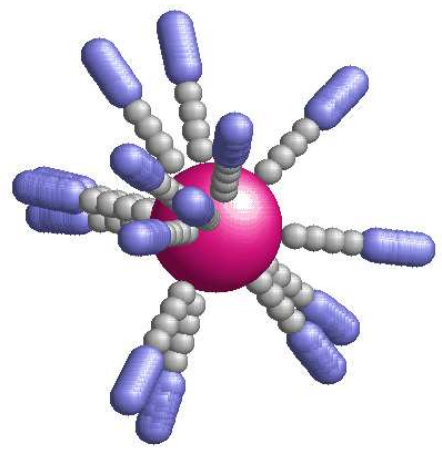

Fig. 1. Generic coarse-grained model for decorated nanoparticle. Central core is shown in pink, spacer - in gray, terminating mesogen unit - as a blue spherocylinder.

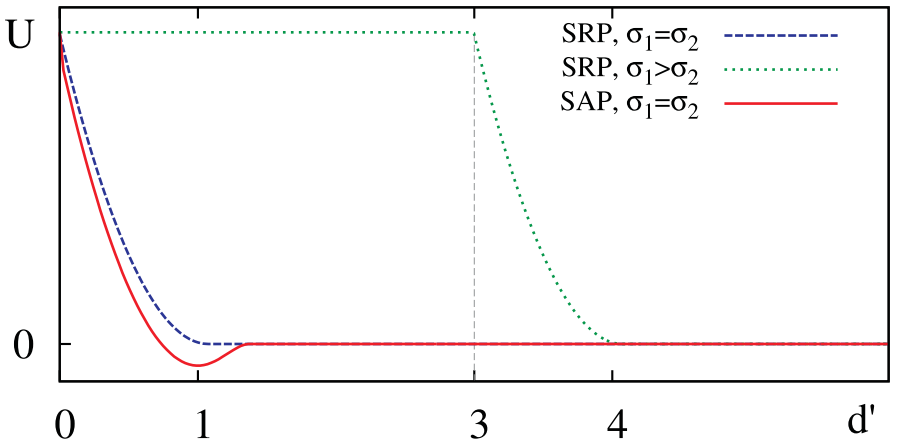

Fig. 2. SRP for the cases of equal and unequal beads dimensions, $\sigma_{1}$ and $\sigma_{2}$, and SAP for the case $\sigma_{1}=\sigma_{2}, d^{\prime}$ is reduced separation between two cores, $U$ - the energy scale (see, respective marking in the figure).

Two general forms for a pairwise interaction potential are used in this study: soft repulsive potential (SRP) and soft attractive potential (SAP). Both are defined in respect to the convex cores of the beads, in a context of Kihara [22]. Namely, the convex core for a spherocylinder is a line that connects the centers of its spherical caps, whereas for the sphere it reduces itself into its center. If $\mathbf{r}_{i j}$ is the vector that connects the centers of $i$ th and $j$ th beads and $\widehat{\mathbf{e}}_{i}$ and $\widehat{\mathbf{e}}_{j}$ are their orientations [these are omitted for spherical bead(s)], then the set $\mathbf{q}_{i j}=\left\{\widehat{\mathbf{e}}_{i}, \widehat{\mathbf{e}}_{j}, \mathbf{r}_{i j}\right\}$ fully characterizes the mutual spatial position of the $i$ th and $j$ th pair. One can define then the shortest distance vector $\mathbf{d}\left(\mathbf{q}_{i j}\right)$, which connects two nearest points between the cores of $i$ th and $j$ th particles. We use the shifted SRP of the following general form

$$
V^{\mathrm{SRP}}= \begin{cases}U, & d^{\prime}\left(\mathbf{q}_{i j}\right)<\sigma_{i j}^{\prime}-1 \\ U\left[1-\left[d^{\prime}\left(\mathbf{q}_{i j}\right)-\sigma_{i j}^{\prime}+1\right]\right]^{2}, & \sigma_{i j}^{\prime}-1 \leqslant d^{\prime}\left(\mathbf{q}_{i j}\right) \leqslant \sigma_{i j}^{\prime}, \\ 0, & d^{\prime}\left(\mathbf{q}_{i j}\right)>\sigma_{i j}^{\prime}\end{cases}
$$

where $d^{\prime}\left(\mathbf{q}_{i j}\right)=d\left(\mathbf{q}_{i j}\right) / \sigma_{0}$ is scaled shortest distance and $\sigma_{i j}^{\prime}=(1 / 2)\left(\sigma_{i}+\sigma_{j}\right) / \sigma_{0}$ is scaled touching dimension. For the chromophore beads $\sigma_{i} \equiv D$. Scaling factor $\sigma_{0}$ has a meaning of a dimension of a soft repulsive shell on the surface of each bead. Namely, for the interacting beads of a same dimension and shape, $\sigma_{0}=\sigma_{i}=\sigma_{j}$, therefore $\sigma_{i j}^{\prime} \equiv 1$ and Eq. (1) reduces to the form with no plateau part (see respective curve in Fig. 2). If $\sigma_{i} \neq \sigma_{j}$, then we choose $\sigma_{0}$ equal to the smallest bead in a system. This renders a large core particle as constantly soft repulsive inside, at $\left(\mathbf{q}_{i j}\right)<\sigma_{i j}^{\prime}-1$, whereas having a shell of thickness $\sigma_{0}$ on its surface, where the repulsion decreases quadratically (again, see, respective curve in Fig. 2). The energy scale is given by a constant $U=70 \cdot 10^{-20} \mathrm{~J}$.

The general expression for the attractive potential can be written as [21, 23, 24]

$$
V^{\mathrm{SAP}}= \begin{cases}U, & d^{\prime}\left(\mathbf{q}_{i j}\right)<\sigma_{i j}^{\prime}-1, \\ U\left\{\left[1-d^{\prime}\left(\mathbf{q}_{i j}\right)-\sigma_{i j}^{\prime}+1\right]^{2}-\varepsilon^{\prime}\left(\mathbf{q}_{i j}\right)\right\}, & \sigma_{i j}^{\prime}-1 \leqslant d^{\prime}\left(\mathbf{q}_{i j}\right) \leqslant \sigma_{i j}^{\prime}, \\ U\left\{\left[1-d^{\prime}\left(\mathbf{q}_{i j}\right)-\sigma_{i j}^{\prime}+1\right]^{2}-\varepsilon^{\prime}\left(\mathbf{q}_{i j}\right)\right. & \\ \left.-\frac{1}{4 \varepsilon^{\prime}\left(\mathbf{q}_{i j}\right)}\left[1-d^{\prime}\left(\mathbf{q}_{i j}\right)-\sigma_{i j}^{\prime}+1\right]^{4}\right\}, & \sigma_{i j}^{\prime} \leqslant d^{\prime}\left(\mathbf{q}_{i j}\right) \leqslant d_{c}^{\prime}, \\ 0, & d^{\prime}\left(\mathbf{q}_{i j}\right)>d_{c}^{\prime},\end{cases}
$$


where the dimensionless configuration dependent well depth $\varepsilon^{\prime}\left(\mathbf{q}_{i j}\right)$ is obtained from the requirement that both the potential and its first derivative vanish at $d_{c}^{\prime}[23]$. This gives the following expression

$$
\varepsilon^{\prime}\left(\mathbf{q}_{i j}\right)=\left\{4\left[U_{a}^{\prime}-5 \varepsilon_{1}^{\prime} P_{2}\left(\widehat{\mathbf{e}}_{i} \cdot \widehat{\mathbf{e}}_{j}\right)-5 \varepsilon_{2}^{\prime}\left(P_{2}\left(\widehat{\mathbf{r}}_{i j} \cdot \widehat{\mathbf{e}}_{i}\right)+P_{2}\left(\widehat{\mathbf{r}}_{i j} \cdot \widehat{\mathbf{e}}_{j}\right)\right)\right]\right\}^{-1}
$$

where $\widehat{\mathbf{r}}_{i j}=\mathbf{r}_{i j} / r_{i j}$ is the unit vector along the vector $\mathbf{r}_{i j}, U_{a}^{\prime}, \varepsilon_{1}^{\prime}$ and $\varepsilon_{2}^{\prime}$ are dimensionless parameters defing the shape of the potential well, $P_{2}(x)=\left(3 x^{2}-1\right) / 2$ is the second Legendre polynomial. Again, the expression (2) is simplified for the case of the same beads, when $\sigma_{i j}^{\prime} \equiv 1$. Otherwise, for the beads of different type, the potential is shifted by $\sigma_{i j}^{\prime}-1$ in respect to $d^{\prime}\left(\mathbf{q}_{i j}\right)$ (see Fig. 2). The use of the potentials (1) and (2) for particular bead-bead interaction in our model is discussed in detail in Sec. 3.

Bonded interactions include harmonic bond stretch and harmonic angle bending terms. Bonding energy per one molecule (decorated nanoparticle) is equal to

$$
V_{b}=\sum_{i=1}^{N_{b}} k_{b}\left(l_{i}-l_{0}\right)^{2}+\sum_{i=1}^{N_{a}} k_{a}\left(\theta_{i}-\theta_{0}\right)^{2},
$$

where $N_{b}$ and $N_{a}$ is the number of bonds and angles in one molecule, respectively. The following force field constants are used: bond stretch constant: $k_{b}=50 \cdot 10^{-20} \mathrm{~J} /(0.1 \mathrm{~nm})^{2}$; bond lengths: $l_{0}=1.49 \mathrm{~nm}$ (core bead-to-first spacer bead), $l_{0}=0.36 \mathrm{~nm}$ (first-to-second spacer beads) and $l_{0}=0.362 \mathrm{~nm}$ (following spacer beads), and $l_{0}=0.298 \mathrm{~nm}$ (last spacer bead-to-the center of the nearest spherical cap of a chromophore); bending energy constant: $k_{a}=50 \cdot 10^{-20} \mathrm{~J} / \mathrm{rad}^{2}$; bending angle: $\theta_{0}=\pi . l_{i}$ and $\theta_{i}$ are instant values for $i$ th bond length and $i$ th pseudo-valent angle, respectively.

Following Ref. [21], to model the photo-isomerisable system the simulation combines both deterministic molecular simulation and stochastic photoisomerisation rules. The photoisomerisation changes trans-tocis and cis-to-trans are defined via their respective probabilities $p_{i}(t \rightarrow c)$ and $p_{i}(c \rightarrow t)$ :

$$
\left\{\begin{array}{l}
p_{i}(t \rightarrow c)=p_{t}\left(\widehat{\mathbf{e}}_{i} \cdot \widehat{\mathbf{i}}^{2}\right. \\
p_{i}(c \rightarrow t)=p_{c}
\end{array}\right.
$$

where $p_{t}$ and $p_{c}$ set the transition rates for the respective processes.

\section{Results}

As already discussed in Sec. 1, the substituted azobenzene chromophores exist that change their dipole moment magnitude $d$ from almost unpolar $(d=1 \mathrm{D})$ trans-isomer to highly polar $(d=5 \mathrm{D})$ cisisomer [13]. The state of all these chromophores can be changed into cis-form by means of the UV illumination, whereas they all will be converted back to the trans-form be means of Vis light $[8,12,25-$ 27]. As the result, the decorated nanoparticles in a polar solvent behave differently in both cases, namely, the colloidal dispersion is formed for the all-cis case and the aggregation is observed for the all-trans state $[15,16]$. The aggregation in the latter case is also enhanced by the liquid crystallinity of trans-isomers.

To reflect these physico-chemical changes on a level of coarse-grained modelling of this study we choose the appropriate potentials for each specific bead-bead pairwise interaction:

- trans-isomer-trans-isomer, trans-wall: SAP potential, Eq. (2);

- cis-isomer-solvent sphere: SAP potential, Eq. (2);

- all other combinations: SRS potential, Eq. (1).

The attractive potential between trans-isomers reflects their liquid crystallinity, whereas the attractive (repulsive) potential between a cis-isomer (trans-isomer) and a solvent bead mimics the condition of a good (poor) solvent condition. The dimensions of the beads have been already provided in Sec. 2, the 

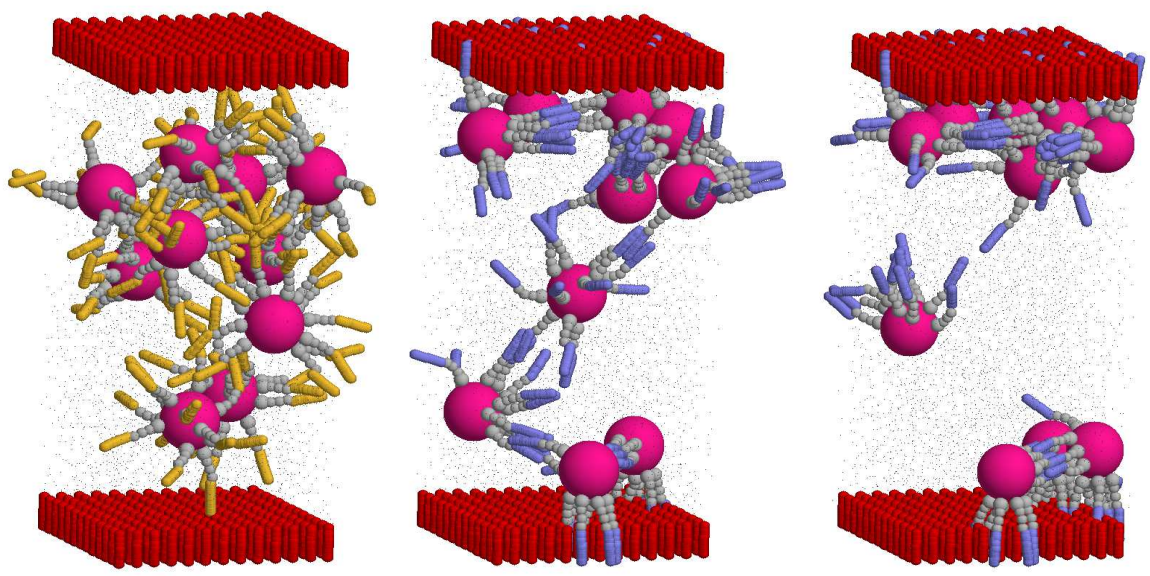

Fig. 3. Snapshots for the all-cis case (left frame) and all-trans case (middle and right frames) at $N_{\text {mol }}=10$. For the latter case both situation of percolation path formation (middle frame) and no percolation path (right frame) are shown).

energy scale is set by $U=70 \cdot 10^{-20} \mathrm{~J}$, whereas dimensionless ratios for the SAP are: $U_{a}^{\prime}=1500 / 70$, $\varepsilon_{1}^{\prime}=120 / 70, \varepsilon_{2}^{\prime}=-120 / 70$ reproducing the "model A" in Ref. [23].

The simulations are performed in the $N V T$ ensemble at $T=400 \mathrm{~K}$ with the periodic boundary conditions along $X$ and $Y$ axes and with the impenetrable elastic walls along to $Z$ axis. The dimensions of the simulation box are: $L_{x}=L_{y}=10 \mathrm{~nm}$ and $L_{z}=20 \mathrm{~nm}$. Each wall is decorated by a ordered layer of immobilised mesogens spaced at the distance of $0.77 \mathrm{~nm}$ apart and all oriented along the $Z$ axis. The layers are introduced to promote adsorption of trans-isomers on each wall. To this purpose the frozen mesogens are chosen of the same type as the trans-isomers but are not photo-isomerisable.

In this study we consider the case of nanoparticles decorated by $N_{\mathrm{ch}}=12$ chains and a number of nanoparticles in a system varies from $N_{\mathrm{mol}}=10$ to 52 to vary their density in a solution. The rest of the simulation box is filled by a solvent until the desired density of $1.5 \mathrm{~g} / \mathrm{cm}^{3}$ is achieved. This is approximately the density at which the self-assembly of decorated nanoparticles have been examined in a concentrated solution (melt) state without illumination $[18,19,20]$. To examine switching of the self-assembly we apply a pulse-like "illumination" which mimics the UV-Vis repeating cycles. This has two main reasons: to examine the reversibility of morphology changes during each cycle and to improve statistics for the averages within each phase of a cycle. We perform 10 cycles, each consisting of 10 ns-long UV phase followed by 10 ns phase of Vis light illumination. Each type of the illumination is modelled by choosing the set of transition probabilities $p_{t}, p_{c}$ in transition rules (5). In particular, during the UV phase we set $p_{t}=1, p_{c}=0$ and during the Vis light phase: $p_{t}=0, p_{c}=1$. These choices lead to quick turning the system into the all-cis and all-trans state, respectively.

What is observed in each phase is illustrated in Fig. 3 for the case of $N_{\mathrm{mol}}=10$. In the all-cis phase (left frame of this figure) no adsoption of the nanoparticles on the walls occured. It is easy to understand, as far as the cis-wall interaction is repulsive (SRP), whereas the cis-solvent is attractive (SAP), therefore the cis-isomers (and, consequently, the nanoparticles) prefer to stay in the bulk. Now, in the bulk, the nanoparticles do not aggregate. This occurs, firstly, due to repulsive cis-cis interaction and, secondly, due to good solvation of each cis-isomer by a solvent. In the all-trans-phase (middle and right frames) the reverse is observed. The adsorption of trans-isomers on the walls occurs due to the attractive trans-wall SAP, resulting in ragging the nanoparticles to both walls. In the bulk, the trans-trans links are formed due to attractive SAP between them. This effect is amplified by the depletion forces between these isomers, due to their poor solubility in a solvent (the trans-solvent interaction is SRP). This may (as seen in a middle frame of Fig. 3) or may not (as in the ight frame of the same figure) lead to the formation of a wall-wall percolating cluster of nanoparticles. 
To analyse the level of aggregation (or, alternatively, clustering) in a system and the existence of a percolation between two walls we introduce a set of related characteristics. As the first step, a connectivity map conn $(i, j)$ is built for each pair $i, j$ of nanoparticles. The pair is considered to be connected if two nanoparticles are linked at least by one chain from both parties. To build $\operatorname{conn}(i, j)$, we perform the loop over all pairs of chromophores $l, m$ (where $l$ belongs to $i$ th and $m$ belongs to $j$ th nanoparticle) and check for their physical crosslinking following a geometric criterion

$$
\left|\left(\widehat{\mathbf{e}}_{l} \cdot \widehat{\mathbf{e}}_{m}\right)\right|>0.966, \text { and } r_{l m}<1.25 D,
$$

which takes into account both the levels of their collinearity and proximity. The second step is, using connectivity matrix conn $(i, j)$, to build for each $i$ th nanoparticle the neighbors list $\mathrm{nn}(i)$ of nanoparticles that are connected to it. Finally, the third step is to split the system into aggregated clusters. In the following algorithm, shown in steps, the clusters are indexed by $m$ and at the end each $i$ th nanoparticle will acquire its host cluster label $\operatorname{clust}(i)$ :

1. initiate $\operatorname{clust}(i):=0$ for all $i$, set first cluster index $m=0$;

2. pick some $i$ th nanoparticle with clust $(i)=0$ randomly;

3. set cluster index $m:=m+1$, assign $\operatorname{clust}(i):=m$, mark $i$ as the cluster $m$ newcomer;

4. loop over the array $\mathrm{nn}(i)$ for each cluster $m$ newcomers adding them to the newcomer list;

5. repeat steps $4-5$ until no newcomers;

6. go to step 2 .

Alternatively, the Hoshen and Koppelmann [28] algorithm can also be used.

Each cluster is characterised by its size $S(m)$, which is equal to the number of nanoparticles it cointains. We analyse the reduced characteristics such as: number of clusters $N_{\text {cls }}$, average cluster size $S_{\mathrm{av}}$, maximum cluster size $S_{\max }$ and maximum cluster span in $Z$-direction (along the normal to the walls surfaces) $Z_{\max }$, defined as

$$
N_{\mathrm{cls}}=\frac{\# \text { of clusters }}{N_{\mathrm{mol}}}, S_{\mathrm{av}}=\frac{\langle S(m)\rangle}{N_{\mathrm{mol}}}, \quad S_{\max }=\frac{\left.S(m)\right|_{\max }}{N_{\operatorname{mol}}}, \quad Z_{\max }=\frac{\text { span in Z }\left.\right|_{\max }}{L_{z}}
$$

In the limiting case of a single percolating network one has: $N_{\mathrm{cls}} \rightarrow 0, S_{\mathrm{av}}=S_{\max }=Z_{\max }=1$, whereas for the completely unconnected nanoparticles: $N_{\mathrm{cls}}=1, S_{\mathrm{av}}, S_{\max }, Z_{\max } \rightarrow 0$, for large enough $N_{\mathrm{mol}}$ and $L_{z}$.

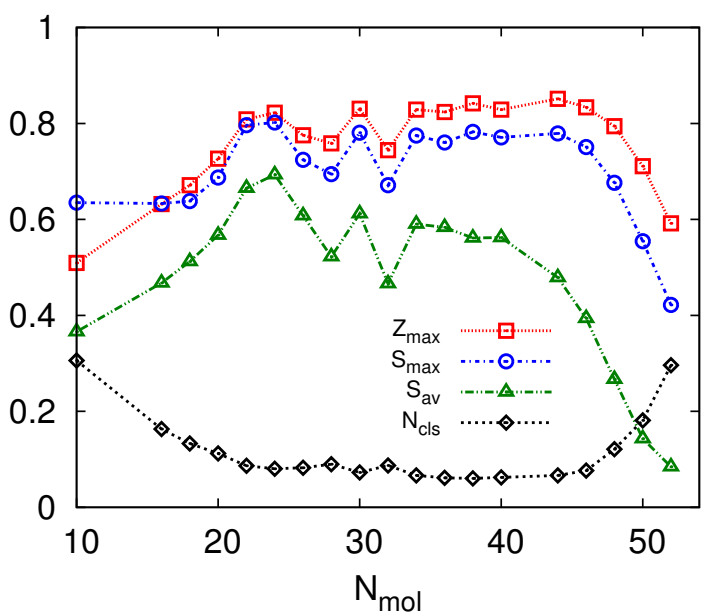

Fig. 4. Reduced number of clusters $N_{\text {cls }}$, average cluster size $S_{\mathrm{av}}$, maximum cluster size $S_{\max }$ and maximum cluster span in $Z$-direction $Z_{\max }$ averaged over the all-trans intervals of a phase trajectory for the different densities of nanoparticles defined by their number $N_{\mathrm{mol}}$.
We average these properties over the all-trans intervals of a phase trajectory only and the result is shown in Fig. 4. One should note a symmetry of all curves: the values for all characteristics at $N_{\text {mol }}=10-20$ are similar to their values at $N_{\text {mol }}=40-50$. That indicates that formation of the percolation cluster is hindered in both depleted and overcrowded cases, whereas there is some optimal interval of $N_{\text {mol }}=20-40$, where the largest maximum cluster (so-called giant component) is able to be formed.

To get more insight on the process of network formation, we analyse also the time evolution of the clustering characteristics shown in Fig. 5. For the case of $N_{\text {cls }}$ (top frame), its value drops from 1 down to $N_{\text {cls }} \approx 0$ each time following the change of the system state from the all-cis to all-trans. The "quality" of response can be estimated from the shape of the $N_{\text {cls }}$ changes which, ideally, should be 

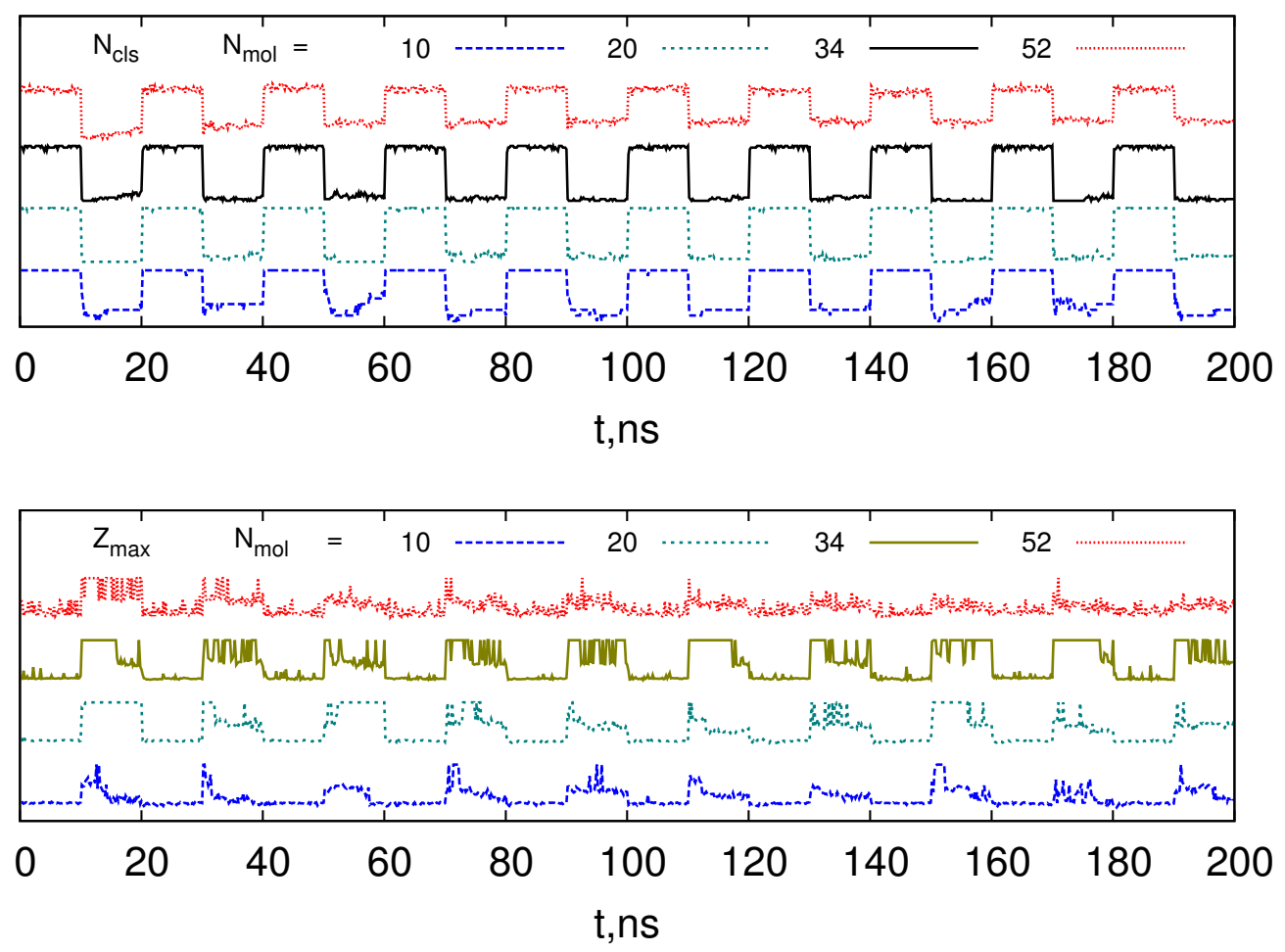

Fig. 5. Time-evolution for the number of clusters $N_{\text {cls }}$ (top frame) and the maximum cluster span $Z_{\max }$ (bottom frame) at various selected $N_{\text {mol }}$ indicated in the figure. Within the time intervals of $0-10 \mathrm{~ns}, 20-30 \mathrm{~ns}$, etc. the system is in the all-cis state, whereas at $10-20 \mathrm{~ns}, 30-40 \mathrm{~ns}$, etc. - in the all-trans state.

square-like and go all the way to the values close to 0 in the all-trans phase. This is so for the case of $N_{\text {mol }}=34$ and, to some extent, at $N_{\text {mol }}=20$, whereas at $N_{\text {mol }}=10$ and 52 the values $N_{\text {cls }} \approx 0$ are not reached indicating a higher degree of clustering. The dynamics of percolation/depercolation can be examined from the time evolution of $Z_{\max }$ shown in the bottom frame of Fig. 5 . As one can see, this property reaches the value 1 (wall percolation) in the all-trans phases most frequently also at $N_{\text {mol }}=34$. This is reflected in the shape of the probability distribution for the maximum cluster span $Z_{\max }$ shown in Fig. 6, which indicates a high peak at $Z_{\max } \rightarrow 1$.

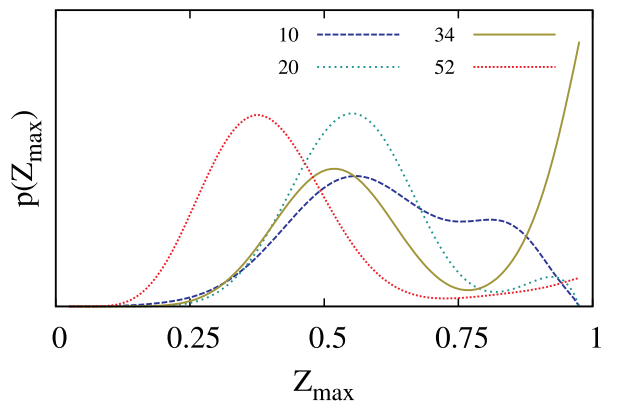

Fig. 6. Histogram for the probability distribution $p\left(Z_{\max }\right)$ at various selected

$N_{\text {mol }}$ (indicated in the figure).
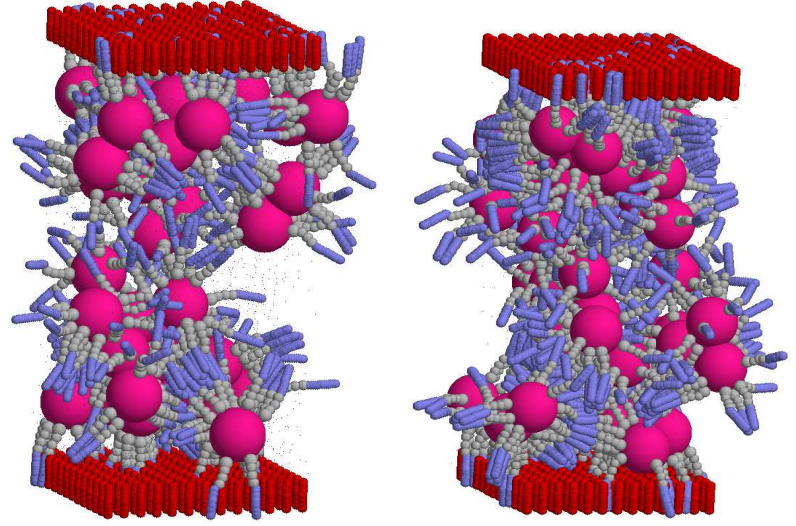

Fig. 7. Snapshots showing the all-trans case at $N_{\mathrm{mol}}=34$ (left frame) and $N_{\mathrm{mol}}=48$ (right frame). 
Based on these simulation studies, we see the following scenario for the percolating network formation in the solution of decorated nanoparticles. At low nanoparticles density, $N_{\text {mol }}<20$, developing of percolation cluster is hindered by its competition with wall adsorption. Indeed, as far as the wall beads are arranged regularly and immobilized, their net interaction with the trans-isomers is stronger than for the trans-isomers between nanoparticles. As the result of a strong adsorption, the number of available nanoparticles to form a percolation cluster in the bulk is reduced. By increasing the nanoparticles density $\left(N_{\mathrm{mol}}=20-40\right)$, the probability of a percolation path formation increases. The question arises why at the further increase of the nanoparticles density, $N_{\text {mol }}=20-40$, formation of the percolating network is hindered again. In our view, the explanantion is that in this case, formation of the network is competing against the microphase separation and, as a consequence, the self-assembly of nanoparticles. This happens in the concentration solution state, where such self-assembly takes place for this type of nanoparticles indeed [18-20]. The self-assembled domains at $N_{\text {mol }}=48$ can be seen clearly in the respective snapshot shown in Fig. 7 (right frame), whereas at $N_{\text {mol }}=34$ their formation is not observed. As the result of a self-assembly, the number of available free arms of each nanopaticle is greatly reduced, affecting the ability to participate in a percolation path formation. This explanation is also supported by the observation made from the Fig. 5, namely, that the maximum cluster span $Z_{\max }$ reaches the value 1 most frequently at the first instances after the system switches from the all-cis to the all-trans state. Here the system "remembers" its dispersed state and quickly forms the network links. As the all-trans state evolves, the microphase self-assembly tendency is brought into action, resulting in oscillatory behaviour for $Z_{\max }$, as seen in Fig. 5. This behaviour is the result of dynamical breakage and reestablishment of the intermolecular links.

\section{Conclusions}

We developed an approach to study the photo-assisted formation of the percolation cluster of nanoparticles contained within a pore with modified walls. The pore is filled by a polar solvent. To enable photo-controllability of the system, the nanoparticles are decorated by polymer chains each terminated by the azobenzene chromophore. The pulse-like illumination which alternates the UV and Vis phases is applied, where the effect of the wavelength affects the rates for the photo-isomerisation in the kinetic equations. The difference in a liquid-crystallinity of trans and cis-isomers of azobenzene, as well as the change of their polarity from the unpolar trans to polar cis is mimicked by a choice of the pairwise potential interactions.

Under UV illumination (all-cis phase) the colloid dispersion is formed with no aggregation. Under Vis light the nanoparticles aggregate as the result of strong trans-trans attraction. The quantitative analysis is performed by evaluation the cluster structure of the system. We found that there is an optimal nanoparticles density interval for the formation of the percolation cluster. When the density is reduced, the adsorption on the pore walls suppresses formation of the percolation cluster. However, when it increases, then the microphase separation of nanoparticles hinders the formation of a single network.

The study is closely related to photo-controllable nanoconductors and opens up the possibility of a future analysis, namely: the influence of the number of chains attached to the nanoparticles, the role of the details of the nanoparticle-wall and nanoparticle-solvent interactions as well as the size of the nanoparticle itself. This will be the subject of a future work.

[1] M. Yokoyama, K. Hosokawa, M. Nogi, and T. Naito, editors. Nanoparticle Technology Handbook. Elsevier, Amsterdam, 2008.

[2] Maria Hepel and Chuan-Jian Zhong, editors. Functional Nanoparticles for Bioanalysis, Nanomedicine, and Bioelectronic Devices Volume 1. American Chemical Society, jan 2012.

Mathematical Modeling and Computing, Vol. 3, No. 1, pp. 33-42 (2016) 
[3] Rafal Klajn, J. Fraser Stoddart, and Bartosz A. Grzybowski. Nanoparticles functionalised with reversible molecular and supramolecular switches. Chemical Society Reviews, 39(6), 2203, 2010.

[4] Roger H. Bisby, Carole Mead, and Christopher G. Morgan. Active uptake of drugs into photosensitive liposomes and rapid release on uv photolysis. Photochemistry and Photobiology, 72(1), 57-61, jul 2000.

[5] Xikui Liu and Ming Jiang. Optical switching of self-assembly: Micellization and micelle-hollow-sphere transition of hydrogen-bonded polymers. Angewandte Chemie International Edition, 45(23), 3846-3850, jun 2006.

[6] C. Raimondo, N. Crivillers, F. Reinders, F. Sander, M. Mayor, and P. Samori. Optically switchable organic field-effect transistors based on photoresponsive gold nanoparticles blended with poly(3-hexylthiophene). Proceedings of the National Academy of Sciences, 109(31), 12375-12380, jul 2012.

[7] V. Faramarzi, C. Raimondo, F. Reinders, M. Mayor, P. Samori, and B. Doudin. Optically switchable molecular device using microsphere based junctions. Appl. Phys. Lett., 99(23), 233104, 2011.

[8] Masumi Ikeda, Naoki Tanifuji, Hidehiro Yamaguchi, Masahiro Irie, and Kenji Matsuda. Photoswitching of conductance of diarylethene-au nanoparticle network. Chemical Communications, (13), 1355, 2007.

[9] Kyong ha Shin and Eun Ju Shin. Photoresponsive azobenzene-modified gold nanoparticle. Bulletin of the Korean Chemical Society, 29(6), 1259-1262, jun 2008.

[10] Corinna Raimondo, Bart Kenens, Federica Reinders, Marcel Mayor, Hiroshi Uji-i, and Paolo Samorì. $\mathrm{Au}$ nanoparticle scaffolds modulating intermolecular interactions among the conjugated azobenzenes chemisorbed on curved surfaces: tuning the kinetics of cis-trans isomerisation. Nanoscale, 7(33), 13836$13839,2015$.

[11] Jian Zhang, James K. Whitesell, and Marye Anne Fox. Photoreactivity of self-assembled monolayers of azobenzene or stilbene derivatives capped on colloidal gold clusters. Chemistry of Materials, 13(7), 2323-2331, jul 2001.

[12] Giustiniano Tiberio, Luca Muccioli, Roberto Berardi, and Claudio Zannoni. How does the trans-cis photoisomerization of azobenzene take place in organic solvents? ChemPhysChem, 11(5), 1018-1028, mar 2010.

[13] Haruhisa Akiyama, Kaoru Tamada, Ju’ichi Nagasawa, Koji Abe, and Takashi Tamaki. Photoreactivity in self-assembled monolayers formed from asymmetric disulfides having para-substituted azobenzenes. The Journal of Physical Chemistry B, 107(1), 130-135, jan 2003.

[14] Abhijit Manna, Peng-Lei Chen, Haruhisa Akiyama, Tian-Xin Wei, Kaoru Tamada, and Wolfgang Knoll. Optimized photoisomerization on gold nanoparticles capped by unsymmetrical azobenzene disulfides. Chemistry of Materials, 15(1), 20-28, jan 2003.

[15] Takeshi Kawai, Satoru Nakamura, Akihiro Sumi, and Takeshi Kondo. Control of dispersion-coagulation behavior of au nanoparticles capped with azobenzene-derivatized alkanethiol in a mixed chloroform-ethanol solvent. Thin Solid Films, 516(24), 8926-8931, oct 2008.

[16] Corinna Raimondo, Federica Reinders, Umut Soydaner, Marcel Mayor, and Paolo Samorì. Light-responsive reversible solvation and precipitation of gold nanoparticles. Chem. Commun., 46(7), 1147-1149, 2010.

[17] Sri Wahyuni Basuki, Viktor Schneider, Thomas Strunskus, Mady Elbahri, and Franz Faupel. Lightcontrolled conductance switching in azobenzene-containing MWCNT-polymer nanocomposites. ACS Appl. Mater. Interfaces, 7(21), 11257-11262, jun 2015.

[18] Jaroslav Ilnytskyi, Juho Lintuvuori, and Mark R. Wilson. Simulation of bulk phases formed by polyphilic liquid crystal dendrimers. Condensed Matter Physics, 13(3), 33001, 2010.

[19] Jaroslav Ilnytskyi. Relation between the grafting density of liquid crystal macromolecule and the symmetry of self-assembled bulk phase: coarse-grained molecular dynamics study. Condensed Matter Physics, 16(4), 43004, 2013.

[20] Arsen Slyusarchuk and Jaroslav Ilnytskyi. Novel morphologies for laterally decorated metaparticles: molecular dynamics simulation. Condensed Matter Physics, 17(4), 44001, dec 2014.

[21] Jaroslav M. Ilnytskyi and Marina Saphiannikova. Reorientation dynamics of chromophores in photosensitive polymers by means of coarse-grained modeling. ChemPhysChem, 3180-3189, sep 2015.

[22] Taro Kihara. Convex molecules in gaseous and crystalline states. In Advances in Chemical Physics, pages 147-188. Wiley-Blackwell, jan 1963. 
[23] Juho S. Lintuvuori and Mark R. Wilson. A new anisotropic soft-core model for the simulation of liquid crystal mesophases. The Journal of Chemical Physics, 128(4), 044906, 2008.

[24] Jaroslav M. Ilnytskyi, Andrij Trokhymchuk, and Martin Schoen. Topological defects around a spherical nanoparticle in nematic liquid crystal: Coarse-grained molecular dynamics simulations. The Journal of Chemical Physics, 141(11), 114903, sep 2014.

[25] Nirmal K. Viswanathan, Dong Yu Kim, Shaoping Bian, John Williams, Wei Liu, Lian Li, Lynne Samuelson, Jayant Kumar, and Sukant K. Tripathy. Surface relief structures on azo polymer films. J. Mater. Chem., 9(9), 1941-1955, 1999.

[26] O. N. Oliveira, L. L. Kumar, and S. K. Tripathy. Surface-relief gratings on azobenzene-containing films. In Z. Sekkat and W. Knoll, editors, Photoreactive Organic Thin Films, pages 429-483. Academic Press, California, 2002.

[27] Tomiki Ikeda. Photomodulation of liquid crystal orientations for photonic applications. Journal of Materials Chemistry, 13(9), 2037, 2003.

[28] J. Hoshen and R. Kopelman. Percolation and cluster distribution. i. cluster multiple labeling technique and critical concentration algorithm. Phys. Rev. B, 14(8), 3438-3445, oct 1976.

\title{
Фотоконтрольована перколяція декорованих наночастинок у порі: комп'ютерна симуляція методом молекулярної динаміки
}

\author{
Ільницький Я. М. ${ }^{1,2,3}$, Слюсарчук А. Ю. ${ }^{2}$, Саф'яннікова М. ${ }^{3}$ \\ ${ }^{1}$ Інститут фізики конденсованих систем НАН Украӥни \\ вул. Свенціцького, 1, 79011, Львів, Україна \\ ${ }^{2}$ Національний університет «Львівсъка політехніка» \\ вул. С. Бандери, 12, 79013, Львів, Україна \\ 3 Ляйбніч-Інститут полімерних досліджень \\ 6 Гое Штр., 01069, Дрезден, Німеччина
}

\begin{abstract}
Використовуючи метод молекулярної динаміки, досліджено формування перколяційного кластера у розчині декорованих наночастинок поміщених у пору. Модель враховує фотоперемикання між неполярними trans та полярними cis-ізомерами азобензину, що приводить до або колоїдної дисперсії або агрегації наночастинок у полярному розчиннику. Динаміка формування перколяційного кластера вивчена шляхом прикладення пульсуючого опромінення. Знайдено, що перколяція між стінками пори залежить від конкуренціх між такими механізмами: агрегація наночастинок, адсорбція наночастинок на стінках пори та мікрофазне розшарування.
\end{abstract}

Ключові слова: наночастинки золота, азобензин, перколяція.

2000 MSC: $82 \mathrm{C} 05,82 \mathrm{C} 43$

УдК: 538.9 